\title{
La parola che salva: per un ritratto di Dante filosofo del linguaggio
}

\author{
Marcello Ciccuto \\ Università di Pisa \\ m.ciccuto@ital.unipi.it
}

\begin{abstract}
In questo saggio si discutono alcuni elementi relativi all'immagine di Dante filosofo del linguaggio, impegnato nel passaggio della sua scrittura da un'implicazione in valori terrestri e umani a significazioni di alta speculazione teologica. Si analizzano quindi, percorrendo criticamente luoghi specifici così del poema come dei tre canti ventiseiesimi delle cantiche, i modi con cui il poeta punta alla riconquista dell'ordo originalis del dire biblico e teologico. Sono i luoghi nei quali Dante mostra di voler rovesciare il rifiuto già platonico della poesia (per il suo dubbio rapporto con la verità) puntando a fare del volgare invece proprio la lingua del sapere filosofico, all'interno del cui sensus literalis traspaiono gli universali. Siamo in presenza di un gigantesco fenomeno di passaggio da una parola umana a una parola 'divina': dentro di esso l'individualità storica e materiale della prima viene comunque salvaguardata, ai fini della realizzazione di quello che possiamo definire il primo esempio di realismo metafisico. Per questo si studia il confrontarsi dantesco con l'intera tradizione poetica 'terrestre' precedente e coeva, sino al modello di Arnaut che rappresenterebbe l'additamento poetico di una via salvationis che prende le mosse dall'umano «andar lacrimando" per procedere verso una lingua che parla "quasi come per se stessa mossa", che accoglie in sé gli universali e viene proposta quindi come la lingua di un nuovo Adamo. L'intero universo sensibile perciò è fatto parlare attraverso la nuova lingua del sacrato poema (nei canti XXIII-XXVI paradisiaci), ora definitivamente consolidata sul modello della scrittura testamentaria da sempre affidata al credere e non più al vedere.
\end{abstract}

Parole chiave: Divina Commedia; filosofia del linguaggio; parola umana; parola divina.

\section{Abstract}

In this essay I discuss some topics about the image of Dante as language philosopher who was concerned with a shifting of his writing from earthly and human values to signifiers of high theological speculation. So I analyze, by tracing critically some specific topics of the poem (together with the three twenty-sixth canti) the ways in which the poet is going to attain the ordo originalis of biblical and theological writing. Here they are the places where Dante shows his purpose of overturning the platonic refusal of poetry - cause of its doubtful connection with truth - while aiming at transforming the volgare into the very language of philosophical knowledge, in whose sensus literalis we can discern universalia. We have here a gigantic phenomenon of shifting from the human word to a divine one: in it, historical and material individuality of the first is going to be defended in order to 
determine what we may define the first pattern of 'metaphysical realism'. So I study the ways in which Dante shares the whole 'earthly' poetical tradition of previous and contemporary times, leading to the example of Arnaut who may represent the poetical key to a via salvationis which starts from the human "andar lacrimando" in order to obtain a language speaking "quasi come per se stesso mosso" and paired to the language of a new Adam. The whole universe of the senses is made speaking through the new language of sacrato poema, at last strenghtened onto the pattern of biblical writing which was eternally trusting more on believing than on seeing.

Keywords: Divina Commedia; philosophy of language; human word; divine word.

Vorrei sottoporre a discussione nel seminario alcuni elementi implicati al percorso seguito da Dante al fine di porsi quale 'filosofo' di un parlare e di uno scrivere che passano, nell'arco di una vita, da un valore tutto terrestre, umano a significazioni di alta speculazione teologica. Ciò che doveva servire a rilevare, per prima cosa, il proposito di risolvere, anzi di sublimare ed elevare gli aspetti contingenti della personale esperienza cognitiva in qualche cosa di assoluto (appunto "quello che non mi puote venire meno»). In parole diverse, si trattava di delineare una forma di responsabilità - e forma poetica del vivere - che garantisse anche alla collettività di passare dall'impegno in pratiche fattuali, mercantili, politiche (o anche dalla semplice avventura dell'esistenza) ${ }^{1}$ a un ordine e a una forma di agire razionale che, pari a quello degli eroi biblici, dei profeti o dei teologi, si identificasse finalmente nell'agire del personaggio che tutto realizza attraverso il processo creativo della parola (nella fattispecie quella della poesia). Rispetto alle condizioni di oscurità e di difficoltà dello stato di partenza, è il sole nuovo del volgare, vale a dire l'emergere linguistico di una poësis volgare la cui forza salvifica tutta ${ }^{2}$ starebbe nella ricerca interna a essa di un rapporto fra sostanza e stile: si potrebbe persino dire di un'eguaglianza fra io e materia a norma di Convivio II IV $4,{ }^{3}$ insomma di

1. Si tratta di porre a testo, da parte di Dante, alcune esperienze produttrici di dubbio, difficoltà di partenza o smarrimenti personali da intendersi quali momenti di perdita del sé, nell'incapacità dell'anima individuale a realizzare, e quindi attualizzare la sostanza tutta umana della propria presenza nel mondo. Di tale condizione di disagio e impotenza iniziali aveva scritto a suo tempo, appoggiandosi in particolare sull'esempio dei sonetti XXXIIIXXXIV del Fiore e sulle parole iniziali del canto primo infernale, John Took nel suo saggio "Towards a life of Dante: ontological anxiety and the salvific function of the word", Italian Studies, n. 59, 2004, p. 3-7. La prospettiva è stata poi incrementata anche da Sebastiano VAlerio, "Lingua, retorica e poetica nel canto XXVI del Paradiso», L'Alighieri, n. 22, 2003, p. 86-87, come anche da Amedeo Marinotti, «Poesia e filosofia nell'ermeneutica dantesca", La rassegna della letteratura italiana, n. 1, 2010, p. 6-7.

2. Persino di già forte riscontro soteriologico, che Dante ha cura di accreditare passo passo in opposizione più e meno esplicita alla negazione del valore cognitivo della poesia sostenuto da larghe parti della cultura ufficiale, specie di area domenicana (san Tommaso, Giovannino da Mantova, Guido Vernani o Giovanni Domenici). Per questo si considerino le osservazioni di John Hollander, «Dante Theologus-Poeta», Dante Studies, n. 118, 2000, p. 266 sg.

3. Cfr. Тоок, «Towards a life of Dante», cit., p. 8. 
adesione integrale della parola a virtù e grazia. ${ }^{4}$ Nel momento in cui l'autorità della parola poetica sostenuta al suo interno dalla Grazia viene a garantire la veridicità di qualsivoglia narrazione, ${ }^{5}$ ecco che il problema della lingua - ma in fondo anche dell'espressione e della retorica e del dire nella sua integralità significante - fa il suo ingresso dentro l'alveo dell'economia ontologica dell'esperienza umana, ${ }^{6}$ dove la più volte richiamata storicità/attualità del personaggio-Dante diventa il segno di un progetto che, nella forma elevatissima dello stilus tragicus e della sua retorica, punta ogni cosa sulla riconquista dell'ordo originalis del dire biblico e teologico. Ed è con questa scelta, fatto notissimo, che si dovrà anche rimediare a un paradosso avvertito da Dante $\mathrm{e}$ reso esplicito nel corso delle sue innumeri riflessioni sulla funzione della lingua volgare all'interno del progetto di emancipazione cognitiva dell'umanità, quel paradosso che si identifica con la constatazione circa l'essere stato scritto il poema sacro per mezzo di una lingua peritura. ${ }^{7}$ Diciamo allora che comincia a configurarsi così il procedere dantesco verso la «serietà terribile» dell'impresa della Commedia (come aveva scritto mirabilmente Contini «la tecnica è in Dante una cosa dell'ordine sacrale, è la via del suo esercizio ascetico, indistinguibile dall'ansia di perfezione»). ${ }^{8}$ Attraverso i passaggi da Ulisse ai poeti dell'effimero e allo scriba dei fissati ai cardini narrativi dei canti ventiseiesimi delle tre cantiche scopriamo perciò figure e funzioni di personaggi che hanno cercato a modo loro di 'trapassare il segno' del valore cognitivo del linguaggio, rendendolo di volta in volta esemplare di un destino; 'torto' nel caso di Ulisse, di valori effimeri intenzionalmente 'senza grazia' per i poeti provenzali e stilnovistici del Purgatorio; di un eloquio - quello di Adamo - che, assumendo invece a valore sommo, addirittura storico la mutevolezza delle cose umane, sana la ferita post-babelica aprendo la via al nuovo poeta-teologo-filosofo che parla e scrive per sostanze perfette, oramai appunto autentico scriba dei.

Ho anticipato in questo discorso preliminare alcuni degli elementi che discuterò più avanti, ma per adesso dovrebbe contare dir questo: forse Dante

4. È fra queste 'pieghe' che riusciamo a riconoscere anche una figura di Dante filosofo più che, come vorrebbe Hollander, theologus-poeta. Basti pensare al trattamento cui l'Epistola a Cangrande sottopone il salmo 113, dove viene a delinearsi un passaggio dall'Esodo storico alla redenzione dell'umanità da parte di Cristo al centro di tutta la storia, alla nostra conversione di umani e quindi alla nostra gloria futura (cfr. allora Hollander, «Dante Theologus-Poeta», cit., p. 275-277). In questo luogo nevralgico della cultura dantesca ci si chiede cioè o si fa vedere come sia possibile stabilire una convenzione in cui un poema narrativo che descrive azioni come se fossero 'storiche' venga a realizzarsi in una narrazione da intendersi appunto a sua volta storicamente vera e reale.

5. Come nel caso della scrittura evangelica e biblica nel suo complesso. A prendere poi il contesto del canto XXIX del Purgatorio, a proposito del numero di ali del grifone della processione dei Seniori nel Paradiso Terrestre, troviamo Dante eleggere insomma l'allegoria del poeti rispetto a quella dei teologi scrivendo "Giovanni è meco e da lui si diparte», e dunque pensando proprio al fatto che Ezechiele ne conta quattro e Giovanni invece sei.

6. Took, «Towards a life of Dante», cit., p. 10.

7. Per questo si veda VAlerio, «Lingua, retorica e poetica», cit., p. 102.

8. Gianfranco Contini (a cura di), Dante Alighieri, Rime, Torino: Einaudi, $1965^{2}$ [1946], p. X. 
ha in animo di rovesciare il principio già platonico (eppoi agostiniano) preso nei rapporti tra filosofia e poesia in quei luoghi del decimo libro della Repubblica dove Socrate critica la poesia proprio per il suo rapporto dubbio con la verità nonché per il suo effetto negativo così sulla vita individuale come specialmente su quella collettiva, sociale (che tanto interessa al poeta Dante). Espulsa allora dalla città ideale, si permetterà alla poesia di rientrare solo a condizione che si sottometta alla filosofia - ed è qui, e specialmente nel corpo del Convivio, che Dante rovescia a favore del volgare questa stessa prospettiva già platonica, facendo del volgare la lingua del sapere filosofico invece che una lingua sottomessa a esso e utile solo ai fini della comunicazione pratica; una lingua dunque incarnata alla filosofia, al sapere e all'esigenza di conoscere.

Se dunque questa lingua ha anche il compito, di cui dicevamo prima, di accreditare nel mondo le nobili menzogne, essa deve avere in sé le caratteristiche della poesia, e cioè contenere in sé un'unione, una fusione artificiale di universale e particolare, deve anzi far trasparire l'universale attraverso il particolare. ${ }^{10}$ Ora, come è ben noto, questa è una caratteristica che nessuna ars possiede, perché filosofia e teologia sono di norma e da sempre superiori alla poesia perché riguardano la conoscenza degli universali; tuttavia il poeta può avocare a sé il compito di capire e di comunicare - con un linguaggio adatto e meglio anche dei filosofi - quanto conti l'attaccamento al particolare, cioè al livello umano, terrestre, fisico che massimamente lo interssa in quanto essere attivo nel mondo. ${ }^{11}$ Allora la Commedia dice la tipologia degli uomini secondo la loro vita e il loro destino in rapporto ai valori eterni, ${ }^{12}$ ed è commedia 'divina' esattamente in linea con questa precisa, ricercata commistione di due elementi altrimenti inconciliabili per definizione, il divino e l'umano - faceva dunque bene Pio Rajna a supporre che Dante avesse usato il titolo commedia quando aveva innanzi le scene infernali, e che in seguito, non potendo mutare il titolo già affermato, avesse cercato di giustificarlo, nell' Epistola a Cangrande. ${ }^{13}$ Quindi potremmo dire che siamo in presenza di un gigantesco fenomeno di passaggio, da una parola umana (poetica, nel caso dantesco) a una parola 'divina'; con Dante che ha inteso presentare il suo mondo terrenostorico non in modo tale che nelle singole figure e nelle storie narrate il carattere terreno fosse soppresso o anche soltanto indebolito, ma anzi agendo al fine di preservare il grado più intenso del loro essere individuale terreno e storico, e da identificarlo in ultimo con la sorte eterna: conservando dunque, anzi ostentando e talora intensificando la sua materialità, e compiendo così la

9. Cfr. allora David Janssens, «Les habits anciens du philosophe. Poésie, philosophie et art d'écrire», Revue philosophique de Louvain, n. 3, 108, 2010, p. 477-479.

10. Ibid., p. 483.

11. Ibid., p. 488.

12. Marinotti, "Poesia e filosofia», cit., p. 6.

13. Del resto, come ricorda lo stesso Marinotti (art. cit., p. 8, nota), fu Bruno Nardi a sostenere l'autenticità dei primi quattro paragrafi dell'Epistola, che sarebbero null'altro che l'anticipo di un commento teologico alla terza cantica impostato sul canone dell'interpretazione allegorica (nel notevolissimo volume suo Il punto sull'epistola a Cangrande, Firenze: Le Monnier, 1960). 
sua immensa idea figurale, quale sarebbe stata poi intuita e perfettamente descritta da Erich Auerbach. ${ }^{14}$ Dicevo poco fa del passaggio, del percorso di un'idea poematica attraverso i canti ventiseiesimi delle tre cantiche; e non posso a questo punto che partire dalla seconda stazione di detto itinerario, stanti le pagine magistrali di Avalle e Padoan sulla funzione di Ulisse. Mi interessa del resto in misura maggiore capire qual è la strategia retorica dell' $e$ locutio dantesca, come si definisce in queste tappe di cui la seconda appunto non vuole affatto negare l'importanza delle esperienze giovanili nel mondo, seppur limitate come si sa, confrontandosi coi magistri Guinizzelli, Guittone, Brunetto Latini, Bonagiunta e Arnaut (vale a dire coloro che avrebbero svolto un ruolo dottrinario nei termini più adatti alla ricostruzione di questo stesso itinerario intellettuale). Conforterebbe il percorso la rima maestro : destro (vv. 2-4), già del Tesoretto "Or si ne va il maestro / per lo camino a destro / pensando duramente / intorno al convenente / de le cose vedute», che porta in campo la precettistica brunettiana ben atteggiata a vademecum professionale recuperando nel contempo gli stessi rimanti incontrati nel canto XXI infernale, dove risuona la vibrata esaltazione del docere da parte di Virgilio a fronte dell'impertinenza di Malacoda («Credi tu, Malacoda, di vedermi / esser venuto, disse 'l mio maestro / sicuro già da tutti i vostri schermi, / senza voler divino e fato destro?»). Cominciamo a capire che qui è in opera una lettura spregiudicata della tradizione lirica tutta, talché si vedrà alla fine che i 'dolci detti' dell'antica poesia umana risulteranno schiacciati dal superiore parlar materno. In più, ancora in questi luoghi, il giro di recuperi guittoniani finisce per allogarsi in un luogo subordinato, non appena il dire di Dante viene a consolidarsi intorno $\mathrm{a}$ un principio di perfezione tecnica che arriverà a proclamare l'eccellenza del trobar ric di Arnaut (non a caso collocato a chiusura del canto), e insieme ad assorbire sia Guittone sia Bonagiunta nella sua generale visione riformata del canone retorico (più ancora che del morale o dottrinario). Dante realizza questo passaggio con una mossa stilistica non ingenua: con una serie di prelievi lessicali e rimici cioè di straordinaria efficacia, quasi sorprendenti per quel molto che nascondono dentro e riescono poi a portare in superficie. Ad esempio, subito dopo il richiamo del v. 7 del sonetto Lo vostro bel saluto, "parlar non posso ché 'n pene io ardo", col v. 18 che suona «rispondi a me che "n sete e 'n foco ardo", c'è l'apostrofe con la quale Guido Guinizzelli si rivolge al pellegrino dell'oltretomba, vv. $16-7$ «O tu che vai, non per esser più tardo, / ma forse reverente, a li altri dopo», utile a riportare all'ideale di gravitas proprio delle sentenze filosofiche del Convivio (IV xxv 24: «a questa etade è necessario d'essere reverente e disideroso di sapere; a questa etade è necessario d'essere rifrenato sì che non transvada»). ${ }^{15}$ Così si comincia a riconvocare

14. Il quale, come è noto, aveva posto in esergo ai suoi Neue Dantestudien - editi una prima volta a Istanbul nel 1944, e quindi a Bern: Francke e come Studi su Dante a Milano: Feltrinelli, 1963 - il motto eracliteo «il carattere dell'uomo è il suo destino».

15. E cfr. dunque, l’attitudine dantesca rispetto all'antico maestro Brunetto Latini («Io non osava scender de la strada / per andar par di lui; ma 'l capo chino / tenea com'uom che 
quell'età di auctores importanti e decisivi, in modo ancora più esplicito con la sequenza rimica parete : sete : rete - dei vv. 20, 22, 24 estratta dal Lapo Gianni di $O$ Morte, della vita privatrice che fu uno dei compagni del vasel di turbanza della giovinezza dantesca. ${ }^{16}$ Non mancano nemmeno rapporti con la cultura laudistica, centrati ad esempio sul lemma sospeso; ma specialmente ricca di precedenti iacoponici è la schera (per cui cfr. la schiera bruna, v. 34, dei lussuriosi a fianco di quella dei sodomiti), sulla quale Dante costruisce l'immagine da bestiario delle formiche che ammusano. ${ }^{17} \mathrm{E}$ ancor più pare contare la schiera bruna in cui Guinizzelli purga il suo vizio, e che è contenta di farsi presta a quella brieve festa, vv. 31 e 34: la stessa rima presta : festa che, guarda caso, è nel sonetto lussurioso di Guido Volvol te levi, vecchia rabbiosa. E ancora: i rimanti canti : davanti : sembianti (vv. 47, 49, 51) sono gli stessi di $A l$ cor gentil rempaira sempre amore e di Veggio negli occhi de la donna mia, ${ }^{18}$ oltreché nel Fiore, XVII o ancora nel Lapo di Donna, se l prego de la mente mia e di Lamentomi di mia disaventura, vv. 5-8: «e dicemi Isperanza: Sta a la dura / non ti cessar per reo sembiante dato, / ché molto amaro frutto si matura / e divien dolce per lungo aspettato». Qui dunque rivolgendosi ai 'sembianti' dei lussuriosi Dante vuol loro 'servire a grato' (altra espressione cavalcantiana e guinizzelliana e guittoniana), ${ }^{19}$ rilevando una forma di gratitudine verso padri e amici, anzi una vera e propria riconoscenza espressa nel momento stesso in cui viene ridefinita l'intelligenza poetica di alcuni di loro, grazie alla quale «anche il cieco vederàm. ${ }^{20}$

La verità di Guido però sta alle sue spalle, «di retro al suo tergo» (v. 66), per cui varrà notare l'espediente tecnico del quasi palindromo RETro-TERghi, a mettere in gioco l'equivoco 'ermafrodito' e la moralità di un Guido che invece, per paradossale contrasto, spera pietosamente nell' innanzi, rassicurato in questo proprio da Dante: «O anime sicure / d'aver, quando che sia, di pace stato" (forma altresì di apparente pudicizia dietro cui si nascondono i lussuriosi, a sua volta aderente alla topica trobadorico-siciliana del celar se si guarda ai vv. 89-90 «se forse a nome vuo saper chi semo, / tempo non è di dire, e non saprei»). Il tutto viene rinforzato dalla scelta dell'immagine dello 'stupido montanaro', vv. 67-68, emblema anche questo di estrazione trobadorica, a significare il biasimo verso chi si mostra d'onni vertù salvaggio. E poi il peccato

reverente vada», Inferno, XV, 43-45), assieme alle varie altre occorrenze segnalate nel bel saggio di Francesco SBerlati, «Maestri e amici nel XXVI del Purgatorio», Studi e problemi di critica testuale, n. 2, 2002, p. 94-96.

16. Ibid., p. 96.

17. Ibid., p. 97.

18. Ibid., p. 99-100.

19. Ibid., p. 102.

20. Ricorderemo allora (e ancora con SBERLATI, ibid., p. 102-103) la rilevanza significante delle rime meco : cieco : reco — «cioè: io, non più cieco, grazie ad amore con me porto la sapienza" - assieme al mondo cieco da cui allontanarsi in forza dell'insegnamento di alcuni maestri che è materia di parte della canzone ciniana a Dante, Avegna ched el maggia più per tempo, vv. 15-16. 
dell'appetito ermafrodito e della sua arsura - altro elemento di lessico iacoponico: ${ }^{21}$ è in gioco l'appetito di fera, integralmente umano, di tutti costoro, figuranti pari alle formiche che si disputano un destino ansioso e avverso; e in gioco è, ovviamente, anche il distaccarsi di Dante da esso, là dove il poeta ha il coraggio di dichiarare, pensoso, di 'pascersi' della contemplazione (a fronte quindi del riguardar pasciuto, v. 103, degli immorali amatori, condividendone amici e volere, compartendone lamenti e canto). ${ }^{22}$

Dante mostra di accorgersi che Guinizzelli lo tiene a caro («Dimmi che è cagion per che dimostri / nel dire e nel guardar d'avermi caro»), dichiarando qui apertamente e ancora una volta l'attaccamento ai dolci detti d'amor quale condizione imprescindibile a un rinnovamento, sia morale che retorico, essendo il fine l'accordare i fatti a' dolci detti: l'accostamento agli antichi rimatori della giovinezza serve a far capire che non il patire di Guido nel fuoco bensì la poesia da lui usata «induca lo fattore a buona misericordia colle dolci parole». ${ }^{23}$ Così tutto questo argomentare sui poeti della terrestre giovinezza dantesca potrà sembrare a questo punto riassumibile nelle parole del Convivio, IV II 15-19:

a me conviene lasciare le dolci rime d'amore le quali sogliono cercare li miei pensieri; e la cagione assegno, perché dico che ciò non è per intendimento di più non rimare d'amore, ma però che ne la donna mia nuovi sembianti sono appariti, li quali m'hanno tolta materia di dire al presente d'amore. ${ }^{24}$

Ecco allora Arnaut: la ragione per cui questi superò tutti gli altri rimatori del suo tempo va ricercata nella 'disponibilità alle lacrime', nella fallacia del dolente spirito. Ma è pure evidente che le rime di Arnaut sono agli occhi di Dante quelle di un poeta che ha tentato di 'soperchiare', di andare cioè 'oltre misura'. Si tratta di un'autentica rivalutazione del trobar arnaldiano, nel senso che questi avrebbe indicato una via verso la salvezza e un diverso modo di dire rispetto al passato; non più insomma l'andare lagrimando ma il puntare alla salvezza: così Guinizzelli intravede l'espiazione della sua colpa attraverso l'andare di Dante "al chiostro / nel quale è Cristo abate del collegio», mentre il 'padre / mio' in bocca a Dante, vv. 97-8 diventa nelle parole di Guido il supremo 'paternostro' (grazie a un vistoso riciclo di un passo del Tesoretto, «né dicer paternostro / in chiesa né nel chiostro", ben avvisato, assieme ad altri elementi utili all'interpretazione di questo passo, da Francesco Sberlati. ${ }^{25}$

21. Sberlati, «Maestri e amici», cit., p. 106.

22. Per il pensoso del v. 100 Sberlati rinvia giustamente a Purgatorio XX, v. 151 e quindi, in evidente anticipo rispetto all'Arnaut purgatoriale del plor e vau cantan, il «vo pascendomi sospiri» del sonetto ciniano a Dante Dante, $i$ ho preso l'abito di doglia.

23. Sberlati, «Maestri e amici», cit., p. 112.

24. Sì che la lascivia del peccato ermafrodito non potrò più trovare posto fra le penne elette che "di retro al dittator sen vanno strette»; né Dante potrà mancare di ricavare da esse un senso di tristizia per via del peccato di quel padre (ibid., p. 116).

25. Ibid., p. 121. 
Dalla passada folor si trascorre immaginativamente, per via di desiderio chiaramente espresso, alla speranza nell'avvenire, puntando verso il chiostro retto da Cristo (e il gioco rimico privilegio : collegio, vv. 127-29 è lo stesso di Falsembiante in Fiore CXI e, unito a dispregio, del XXIII infernale, là dove scatta la requisitoria sui valori disattesi da parte di Catalano dei Malavolti), verso insomma "la peschiera ch'è tranquilla e pura» di Par. V, 100. Infine Guinizzelli, dopo aver parlato di foco ardente, dopo aver fatto rimare fra loro loco e foco e parecchio altro, ${ }^{26}$ si rifugia nell'acqua "come il pesce andando al fondo", v. 135. È rilevante constatare come dietro questa indicazione di movimento stia un'immagine pescata - è proprio il caso di dirlo - da Meraviglioso beato di Guittone, al pari della coerenza dire : disire che è pressoché tipica di parecchie canzoni dell'aretino: perché Guittone è presentissimo negli otto versi del dire di Arnaut, a cominciare da quel tan mabellis corrispondente al tanto abellire della canzone Omo fallito, plen de van penseri e di $O$ tu, lassomo, che ti dai per amore ${ }^{27}$ perché proprio al sermone di Arnaut corrispondono misure tutte guittoniane, intendendo qui Dante privilegiare una prospettiva unitaria tra lirica in lingua d'oc e poesia in lingua del sì. Ed ecco dunque il punto: la speranza di cui Arnaut è fatto interprete significa aprire a un avvenire (denan) denso di joi, la follia del passato recando in sé solo esperienza di falsità e di dolore; e lo stesso poeta provenzale potrà dirsi convinto di aver partorito col suo 'parlar materno' una generazione di dicitori in rima che esibisce il tradimento della passione amorosa riscattandola, indirizzandola verso un' affettività spirituale molto più complessa.

Sappiamo che questo discorrere sulla lingua poetica riemerge con prepotenza giusto nell'altro canto XXVI. Ancora una situazione di partenza che è di smarrimento («Mentr'io dubbiava per lo viso spento /de la fulgida fiamma che lo spense/uscì un spiro che mi fece attento») e ancora un richiamo alla figura di Beatrice con esibite allusioni al linguaggio del periodo giovanile, che, fatto più che noto, ${ }^{28}$ viene posto a confronto, alla prova della caritas (si pongano appunto in linea Exodus 33, 19 «Ego ostendam omne bonum tibi» e il versi attorno al 42 «Sternel la voce del verace autore / che dice a Moisè, di sé parlando: / Io ti farò vedere ogne valore»). Ora è il momento del trapasso verso la scrittura teologica, pari a quella biblica appunto, dove allo scriba dei è possibile parlare con una lingua "quasi come per se stessa mossa»; ${ }^{29}$ ed è anche il momento nel quale, riepilogando altre esperienze di parola, Dante più che altrove si trattiene sulla questione, centralissima, della scrittura poetica: «Lo ben che fa contenta questa corte / Alfa e $\mathrm{O}$ è di quanta scrittura / mi legge Amore o lievemente o forte». Come dire che in questi canti è materia di scrittura che si vede risalire alle fonti dell'essere e del dire. Sarà quindi la funzione del personaggio-Adamo (che - lo abbiamo già accennato - deve servire a

26. Cfr. ancora ibid., p. 123-124.

27. Come appunto notato ibid., p. 127.

28. Cfr. VAlerio, «Lingua, retorica e poetica», cit., p. 88.

29. Ibid., p. 90-96. 
giustificare il paradosso di un testo sacro scritto in una lingua peritura come il volgare) quella di introdurre una concezione linguistica almeno in parte rivoluzionaria rispetto a quanto Dante aveva affermato in precedenza, a proposito del primiloquium, cioè dell'esprimersi con una lingua creata assieme ad Adamo e consegnata solo all'uomo fra tutte le creature, e cioè l'ebraico. Così le parole dei vv. 130-38:

Opera naturale è ch'uom favella ma così o così, natura lascia poi fare a voi secondo che v'abbella pria ch'i' scendessi a l'infernale ambascia I s'appellava in terra il sommo bene onde vien la letizia che mi fascia e El si chiamò poi: e ciò convene, ché l'uso d'i mortali è come fronda in ramo, che sen va e altra vene

spiegano subito perché tanto e tanto a lungo Dante si sia intrattenuto a parlare delle componenti 'mortali' della lingua poetica sua e di altri: ora non si tratta più di tenere in conto la mutabilità della lingua tipicamente umana, l"uso moderno' del v. 113 - che non viene comunque rinnegato - ma di approdare alla creazione di una lingua cognitiva capace di abbracciare, più propriamente di accogliere gli universali: lingua che non si può attribuire dunque a un solo autore, perché è piuttosto il frutto di una maturazione poietica, è vissuta e vive di precisi antecedenti. ${ }^{30} \mathrm{Si}$ chiuderà appunto qui il cerchio di un progetto che doveva portare a riconoscere nel volgare la lingua perfetta e fissata a concetti eterni di verità e di carità (con Dante filosofo che arriva a compimento della sua ricerca di verità e di sostanze dell'essere e che può finalmente esprimere con una lingua adatta). E allora, diciamolo: è Dante che vuole diventare il nuovo Adamo, restituendo a Dio, in poesia, le voci popolari e la teologia, la grande poesia e le sacre scritture, il suo passato e il suo presente, una miscela più e meno equilibrata di caritas, poesia stilnovistica, retorica $\mathrm{e}$ linguistica. ${ }^{31}$

Se trascorriamo a questo punto direttamente al discorso del primo padre, nel XXVI paradisiaco, ci si accorge come esso punti subito dritto a rivendicare la componente terrena, mondana del sole nuovo, con un aggancio al verbo abbellare che abbiamo già visto centrale nelle poche parole di Arnaut - Tan mabellis vostre cortes deman - e che nascondendo il piacere interno all'uso moderno diventa davvero decisivo dell'ampio ragionamento dantesco sul dire poetico. ${ }^{32}$ Ripetiamo allora:

30. Ibid., p. 103.

31. Che è quanto più ampiamente e dettagliatamente rilevato ibid., p. 104.

32. Molto chiaro su questo il saggio di Edoardo Ferrario, «Il linguaggio nel XXVI canto del Paradiso", in Miscellanea di studi in onore di Aurelio Roncaglia, I, Modena: Mucchi, 1989, p. $559-579$. 
La lingua ch'io parlai fu tutta spenta innanzi che a l'ovra inconsummabile fosse la gente di Nembrot attenta; ché nullo effetto mai razionabile, per lo piacere uman che rinovella seguendo il cielo, sempre fu durabile. Opera naturale è ch'uom favella; ma così o così, natura lascia poi fare a voi secondo che v'abbella. Pria ch'i'scendessi a l'infernale ambascia, I s'appellava in terra il sommo bene onde vien la letizia che mi fascia; e El si chiamò poi: e ciò convene, ché l'uso d'i mortali è come fronda in ramo, che sen va e altra vene

Ad anticipare alcuni elementi della discussione, vorrei rilevare il momento il cui Dante vede un quarto lume accanto a quelli che 'rappresentano' gli apostoli Pietro, Giacomo e Giovanni; il poeta esprime un disio di parlare a chi letteralmente 'vede' questa sua voglia, «verace speglio/che fa di sé pareglio e l'altre cose,/e nulla face lui di sé pareglio», riflettendo cioè identica ciascuna delle cose, nessuna delle quali può fare lo stesso di lui). Quindi la risposta di Adamo risulta preceduta da una similitudine che mette in parallelo il moto del suo spirito nel venire alla parola e l'eccitazione muscolare di un cavallo che 'broglia', si agita sì da manifestare in forma di forte evidentia il suo affetto. Chiariremo meglio più avanti il senso di questo confronto. ${ }^{33}$ Per adesso conta dire invece e isolatamente che la questione posta da Dante e Adamo risponde all'ultima delle sei quaestiones relative alla prima espressione della parola umana Ibid. (così in De vulgari eloquentia I IV 1), dove solo l'uomo ha bisogno della parola, segno della sua differenza e della sua identità e - fra le obiezioni possibili nel passo del trattato - segno anche della sua peribilità: sarà perciò questo specifico valore di lingua peritura che Dante dovrà giustificare, legando il linguaggio al sentirsi, alla condizione umana pura e semplice. Sentirsi uomo e parlare saranno insomma la stessa cosa. ${ }^{34}$

Come corollario a detta notazione Dante introduce poi addirittura una sconfessione del De vulgari eloquentia riguardo a una lingua originariamente unica e durevole per natura, pensando cioè alla nozione di grammatica, di una lingua che, regolata per mezzo dell'arte, ripristina artificialmente, attraverso una convenzione, la condizione di originaria incorruttibilità. Ciò che quindi rappresenta lo sfondo di legittimazione del volgare illustre, ${ }^{35}$ che viene a significare sostanzialmente questo: usando i criteri della convenienza e del piacere, il pensiero del poeta si sposta da un concetto astratto di unità linguistica ori-

33. Ma per questo e quanto anticipato si veda ancora ibid., p. 560.

34. Ibid., p. 565.

35. Ibid., p. 568. 
ginaria alla rilevanza dell' uso dei mortali, all'essenza del linguaggio come bisogno e piacere dell'uomo (finendo per privilegiare quindi non l'ebraico ma una lingua individuale creata dal primo uomo stesso); ${ }^{36}$ tutto quel che vale insomma tornare a dire dello stato di insufficienza, finitudine, indigenza quali condizioni fondamentali ad accreditare l'usus (termine ripetuto per ben due volte, ai vv. 136-37, con una mossa che spinge a ricordare come nel I del Convivio proprio l'uso veniva già contrapposto a regola, con l'immagine del latino 'che seguita arte' e il volgare che 'seguita uso'). Nel trattato linguistico le parole mamma e babbo erano state dichiarate inammissibili al volgare aulico perché d'uso puerile; e proprio queste due parole rimaste nel cribro del dire tragico ricorrono addirittura nell'espressione che, nell'ultimo lembo di inferno, definisce la lingua in cui è scritta la Commedia: la lingua che chiama mamma $e$ babbo, la stessa che nelle successive cantiche dirà parole antitragiche come il pappo e il dindi e nanna e barba e quella donna Berta che nel trattato latino serviva a esemplificare nientemeno che il grado insipidus del parlare. ${ }^{37}$ Come si vede, Dante avrebbe cambiato idea rispetto al trattato linguistico, e molto sembra impostare sul principio tutto terreno del piacere (rivendicato addirittura da Virgilio quando fa Dante signore di se stesso dopo averlo condotto oltre i grandi poeti dell'uso moderno, nel contesto del canto XXVII: «Tratto t'ho qui con ingegno e con arte;/lo tuo piacere omai prendi per duce», che corrisponde poi all'abellare del miglior fabbro del parlar materno)..$^{38}$

L'episodio di Adamo rappresenta a questo punto un'autogiustificazione della Commedia e del suo pensiero linguistico. E il fatto che sia Arnaut sia Adamo vadano incontro al desiderio, anzi proprio al piacere del poeta, uscendo da ciò che li copre e li avvolge (il fuoco che li affina), equivale al far incontrare una condizione di pura intelligibilità, di letizia sovrasensibile, con l'estremo opposto della sensualità che muove il piacere animale - il cavallo che broglia, appunto, di cui abbiamo accennato poco fa. I paragoni animali di Adamo e di Guinizzelli (ricorderemo per un attimo solo quest'ultimo che sparisce come un pesce verso le profondità marine) localizzeranno allora $\mathrm{i}$ confini entro i quali la parola umana cessa di essere utile; così le anime dell'aldilà saranno coerentemente aldilà del bisogno, dell'uso del linguaggio che invece caratterizza una moltitudine di 'espressioni' all'interno del poema, sotto l'egida dell'immagine di Paolo e Francesca richiamati dall'affettuoso grido' del mortale visitatore quali colombe dal disio, con l'evento di storicità e finitudine che torna col celebre attacco «Siede la terra dove nata fui...». ${ }^{39}$ Invero già prima la figura di Virgilio, colui che per lungo silenzio parea fioco, serve a indicare un evento esemplare di individualità e storicità umana, segnato da confini fattuali, naturali, geografici, astronomici di puntuale esattezza (che vedremo come tornino nella sostanza più accesa del XXIII del Paradiso); e se la voce I è intro-

36. Ibid., p. 568-570.

37. Ibid., p. 571.

38. Ibid., p. 572.

39. Ibid., p. 573-574. 
dotta come cifra linguistica della singolarità storica di Adamo, lo stesso par capitare nel canto XXVII dell'Inferno, con Virgilio sorpreso in un'espressione che diremmo simile: Istra ten va, più non t'adizzo, espressione fatta di comica verba, quindi massima espressione di particolarità ai limiti dell'idioletto e del non senso, costruita sullo stesso modello e tipo di Raphel maì amecche zabì almi o di Pape satan, pape satan aleppe. Dante dunque scandisce precisa equazione fra uso della parola degli uomini e loro soggiorno sulla terra: Lombardo fui e fu' chiamato Marco; Siena mi fe, disfecemi Maremma; voi cittadini mi chiamaste Ciacco, ecc. ${ }^{40}$

$\mathrm{E}$ evidente anche in base a questi soli elementi che la parola viene ora richiamata da Dante come segno di finitudine, proprio nel momento in cui detta parola vede trasporre le sue funzioni dalla condizione di finitezza che ha retto il passato e l'attraversamento infernale all'investitura espressiva degli universalia. Ogni evento di linguaggio viene d'ora in avanti impiegato a ridisegnare un fenomeno di singolarità e di senso, di storicità forte, a formare poi la grande polifonia delle voci del poema: Chiamato fui di là Ugo Ciappetta - Stazio la gente ancor di là mi noma — Folco mi disse quella gente a cui fu noto il nome mio. Ma Dante fa ancor di più, perché si prova a tendere il verso fino al limite della coincidenza del dato linguistico e del significato esistenziale; ed è proprio un atto di articolazione linguistica, ritratto nell'emergere del suo improvviso bisogno o, come abbiamo già detto, tutto umano piacere. Così prendono forza anche espressioni regredite a puro suono, a gestualità ritmicomimica (le diverse lingue, le orribili favelle, il suon di man con elle, l'oscena cennamella del duca Malacoda, le fiche della bestia di Pistoia...), e altri nonsensi eloquentissimi: lo sputo parlante del serpente-uomo, l'inno fatto di bolle d'aria gorgogliato nella strozza dagli accidiosi, le parole-lacrime di Francesca e di Ciacco, il linguaggio vegetale di Pier della Vigna e di altri uomini lacerati come tizzoni verdi che scoppiettano nel fuoco, il muggire e rugghiare delle fiamme e il maggior corno che crolla come vento - ogni espressione, anche la meno 'verbalizzabile', viene riconvertita in fenomeno del senso, operando una sorta di apertura della lingua alla totalità del significare. Si susseguono quindi anime odorose, astri, arpe, orologi, gemme, arcobaleni, le luci che vanno e vengono su e giù la scala d'oro di Saturno, i pesci che emergono dalla limpida peschiera di Mercurio per pasturarsi di parole umane, le minuzie che nella galassia di Marte vanno in alto e in basso, come polvere nel raggio di sole filtrato in una stanza e incontrandosi, scintillano per poi tintinnare come corde di una giga tirate e allentate dalla mano di Dio a formare un'unica melodia; e poi Pier Damiani che esce dalla pura luce che lo occulta ed esprime la sua voglia di compiacere alla parola mettendosi a ruotare su se stesso come una mola; le anime beate del cielo di Giove, uccelli che si riuniscono a stormo e cantando diventano essi stessi le parole del loro canto, e quindi si arrestano e mostrano la scrittura sorta dalla costellazione dei loro corpi: Diligite iustitiam 
qui iudicatis terram; e lo spettacolo della parola umana offerto al visitatore da questa conformazione di corpi alfabetici prima formerà l'allegoria di un giglio, poi animerà il traslato di un'aquila che fa suonare la voce dicendo io e mio.

Ecco, la parola nella Commedia è il miracolo del senso umano, che risuona da un oltre che si tende ai limiti del suo essere negli elementi biologici e fisici, nei fenomeni cosmici originari, nelle formazioni primitive della materia: "così che il cigolare di un stizzo verde messo al fuoco, un gorgoglio di bolle sulla superficie dell'acqua, la polvere in un raggio di sole, il mormorìo di un fiume, il ruotare di una mola, il crepitare di una fiamma, la formazione dell'arcobaleno, il brillare di un astro, possono ricostituirsi in fenomeno di storicità umana: il miracolo di un "poema sacro / al quale ha posto mano e cielo e terra"». ${ }^{41}$ Questo spiega d'altronde alcuni aspetti sostanziali della compagine dei canti XXIII-XXIV paradisiaci, che si apre su un natural dimostramento quasi di estrazione dalla antica fisica guinizzelliana e affezione per l'umano (in mezzo ad amate fronde, dolci nati, aspetti disiati, ardente affetto), perché sta per essere messa in scena la teologia dell'incarnazione, nelle forme di una latenza/presenza del divino dentro l'umano e il terrestre (così del resto già nel XXI: «Luce divina sopra me s'appunta/penetrando per questa in ch'io m'inventro/la cui virtù, col mio veder congiunta/mi leva sopra me tanto, ch'i' veggio/la somma essenza de la quale è munta»). ${ }^{42}$ Il canto XXIII, diviso in due parti che si corrispondono, deve rappresentare una natura prima umana e quindi, in corso di trasformazione, divina: il mondo della pura visibilità o consistenza materiale della luce (nel bel giardino, nell'aiuola corrispondente al trionfo della Chiesa sulla terra) che ora si confronta con quello celeste che è 'turba di splendori' al limite dell'invisibilità e dell'immaterialità. L'attesa del sole su cui si apre il XXIII varrà proprio attesa di un sole (una luce) diverso da quello terrestre, invisibile agli occhi degli umani e solo oggetto di speranza e di desiderio - e analogamente un oggetto di consistenza terrestre, la Luna/Trivia che ride tra le ninfe etterne, è quello che ci dà la misura-figura simbolo di questo momento di passaggio da una realtà reale a una realtà che contiene in sé valori-sustanzie e che le esibisce («e che è ridere se non una corruscazione de la dilettazione de l'anima, cioè uno lume apparente di fuori secondo sta dentro?» è detto nel Convivio). ${ }^{43}$ Così il canto si chiude su un'altra e vistosissima opposizione tra valori umani e terrestri: "Quivi si vive e gode del tesoro/che s'acquistò piagnendo ne lo essilio [...] e con l'antico e col novo concilio" - col ricordo dunque di ben diverso tesoro tutto umano che era stato quello raccomandato, in verità blasfemamente, da Brunetto Latini. ${ }^{44}$

41. Ibid., p. 578.

42. Per questo e quanto segue rinvio al mio saggio «Le luminose sostanze dell'essere. Sacralità della scrittura dantesca nei canti XXIII-XXIV del Paradiso», in c. s. sulla rivista Tenzone.

43. Convivio III viII 11-12.

44. E cfr. allora la lettura di Marcello Ciccuto, «Premessa al Tesoretto di Brunetto Latini», in ID., Il restauro dell' "Intelligenza» e altri studi dugenteschi, Pisa: Giardini, 1985, p. 141-158. 
Qui, nel momento di passaggio dall'umano sentire a fatti di fede, la parola di Dante dice che si sta smaterializzando per acquisire - evidente paradosso - una consistenza sì ma immateriale, per un poema sacro e sacrato in cui la scrittura, al pari della divina favella delle due Scritture, è diventata aderente alla verità vera e sostanziale. È il rapporto fra teologia e retorica umana, fra parola e verità; per cui Dante, ancora, arriva a ridefinire su nuove basi la logica dichiarativa che regge le artes o forse insomma tutto l'agire umano, con la citazione di pressoché tutte le risorse degli argomenti umani, tutto l'umanamente predicabile, professioni, arti e mestieri, artigianato, tecnologia e misurazione del tempo, le comete del mondo celeste e la scrittura, la pittura e il mondo dell'università e delle armi, la zecca, i codici e ancora la scrittura al v. 93, la liturgia, il mondo feudale, la cancelleria, l'azione del fuoco. C’è persino un raffronto fra grammatica razionale e grammatica divina: Dante si premura cioè di dire per ben due volte che è la Grazia a farlo parlare (vv. 58-60 e 118-120), lui eletto, scelto al fine di completare quel processo avvisato solo programmaticamente in Inferno II, 31-33, di realizzare insomma il percorso di Enea in quanto uomo e acquisire il verace stilo di san Paolo in quanto scrittore-poeta.

La scrittura del poema si sta equilibrando allora sul modello della scrittura testamentaria, affidandosi al credere e non più al vedere. E ritorna guarda caso alla più elementare delle scienze, a quella fondativa del dire, la grammatica, che ora offre solo figure logiche come il plurale e il singolare, sono ed este, ai vv. 139-141; tant'è che nella risposta di Pietro a Beatrice Dante inverte consapevolmente, con tante figure di usteron-proteron, il processo di percezione linguistica dell'universo. ${ }^{45} \mathrm{Si}$ arriva all'enunciazione del Credo, dove ancora non si persegue un ordine logico ma uno più elementare e profondo ispirato dalla Grazia, appunto: a un primo contenuto fisico e metafisico della fede ne segue uno che li trascende entrambi, imperniato sulla Rivelazione; poi la terzina finale iscrive l'emozione in fiamma e luce che sono ben distanti da quelle che avvolgevano Ulisse o Arnaut: «e come stella in cielo in me scintilla». Così l'io terrestre è diventato stella, considerate le stelle la manifestazione del Vero, e la scrittura che lo dice una forma assoluta, totalizzante del verbo divino, proiettata mirabilmente sullo schermo della galassia: «e come stella in cielo il ver si vide» (Par. XXVIII, 87), un vero che la scrittura poetica ha riportato ai significati primordiali e più elevati che si identificano con la vita stessa di tutto il cosmo.

45. Per questo e altro vale la lettura di Mario Pazzaglia, «Il canto XXIV del Paradiso», in ID., L'armonia come fine. Conferenze e studi danteschi, Bologna: Zanichelli, 1989, p. 196 sg. 